\title{
Numerical simulation of armored vehicles subjected to undercarriage landmine blasts
}

\author{
A. Erdik ${ }^{2}$ - S. A. Kilic ${ }^{1}$ - N. Kilic ${ }^{2}$ - S. Bedir ${ }^{2}$
}

Received: 2 October 2013 / Revised: 3 May 2015 / Accepted: 5 May 2015 / Published online: 27 May 2015

(c) The Author(s) 2015. This article is published with open access at Springerlink.com

\begin{abstract}
Landmine threats play a crucial role in the design of armored personnel carriers. Therefore, a reliable blast simulation methodology is valuable to the vehicle design development process. The first part of this study presents a parametric approach for the quantification of the important factors such as the incident overpressure, the reflected overpressure, the incident impulse, and the reflected impulse for the blast simulations that employ the Arbitrary LagrangianEulerian formulation. The effects of mesh resolution, mesh topology, and fluid-structure interaction (FSI) parameters are discussed. The simulation results are compared with the calculations of the more established CONventional WEaPons (CONWEP) approach based on the available experimental data. The initial findings show that the spherical topology provides advantages over the Cartesian mesh domains. Furthermore, the FSI parameters play an important role when coarse Lagrangian finite elements are coupled with fine Eulerian elements at the interface. The optimum mesh topology and the mesh resolution of the parametric study are then used in the landmine blast simulation. The second part of the study presents the experimental blast response of an armored vehicle subjected to a landmine explosion under the front left wheel in accordance with the NATO AEP-55 Standard. The results of the simulations show good agreement with the experimental measurements.
\end{abstract}

Communicated by W. G. Proud.

S. A. Kilic

skilic@boun.edu.tr

1 Department of Civil Engineering, Bogazici University, 34342 Bebek, Istanbul, Turkey

2 OTOKAR Otomotiv ve Savunma Sanayi A. S., Ataturk Cd. No:9, 54580 Arifiye, Sakarya, Turkey
Keywords Landmine blast · Mine Resistant Ambush Protected vehicle - Arbitrary Lagrangian-Eulerian technique - CONWEP blast loading - LS-Dyna code . Spherical topology $\cdot$ Cartesian topology

\section{Introduction}

Undercarriage landmine blasts cause a significant threat to occupant safety in armored personnel carriers. The blast wave interaction with armored plates in the undercarriage is an important factor in the design process.

Experimental studies provide valuable insight to the performance of armored vehicles subjected to landmine blast. Some measures of performance include the resistance of the undercarriage against tearing of the armor plates, failure of the structural welds, and high accelerations of the footrest plate used by the occupants. Landmine blast experiments that involve the testing of the full vehicle are costly and time consuming, while numerical simulations provide a faster alternative to measure the vehicle performance under blast loads. The blast resistant undercarriage armor design is an iterative process that is shaped by the successive use of numerical simulations.

There are two major approaches for modeling blast loads. The first approach involves the use of empirical equations obtained from blast experiments. This is referred to as the CONWEP method (CONventional WEaPons). This technique is suitable for simulating structural members directly exposed to the blast wave, without any obstructions or shadowing effects. The second approach is the Arbitrary Lagrangian-Eulerian (ALE) technique that requires the modeling of the surrounding air with a volumetric mesh around the target structure. It allows the application of the NavierStokes fluid dynamics equations for simulating the blast wave 
propagation. The coupling algorithm provides the interaction of the blast wave with the target structure. However, the ALE simulations lead to an increase in computational cost when compared with the CONWEP method. Furthermore, calibration of the ALE parameters is a time consuming process.

The first part of the study presents the calibration of the ALE approach by comparing the overpressure and impulse results with the CONWEP method for a target flat plate directly facing an explosive charge. The topology of the surrounding air is modeled using a uniform Cartesian mesh as well as a spherical mesh.

The advantage of the spherical topology is that the flow of the mesh lines is perpendicular to the direction of the blast wave propagation for a spherical charge. This type of mesh topology yields a higher level of accuracy for the ALE advection algorithm because the close-in shape of the blast wave is similar to the geometry of the charge. However, it should be noted that the blast wave formation will not be best represented with a spherical mesh topology for nonspherical shaped charges in the close-in range. The increase in simulation accuracy due to the employment of higher mesh resolutions is illustrated for both the Cartesian and the spherical topologies.

The second part of the study presents the experimental results for a full-scale blast test of an armored personnel carrier subjected to undercarriage landmine explosion. A landmine with a cylindrical geometry is encased in a rectangular steel pot planted under the front left wheel of the vehicle in accordance with the NATO AEP-55 Standard [1]. Displacement, velocity and acceleration data on the crew compartment are collected. The experimental results are compared with the numerical LS-Dyna simulation employing the ALE method. The optimum simulation parameters obtained from the flat plate study are used in the ALE simulation of the landmine explosion. The spherical mesh topology is employed for the air surrounding the vehicle. It is evident that the cylindrical shape of the charge and the blast wave reflections from the vehicle undercarriage result in a non-spherical blast wave formation for the close-in range in the vicinity of the front wheel where the detonation takes place. However, for the spherical mesh resolution used in the numerical study the computed displacements in the cabin nearest to the blast location are in good agreement with the experimental measurements.

\section{Literature review}

The most common mesh topology used in ALE type of blast simulations is the Cartesian geometry in which the Eulerian domain for the surrounding air is modeled with hexahedral elements with orthogonal mesh lines. However, there are a limited number of studies in the literature with spheri- cal Eulerian mesh topologies. Chafi et al. [2] investigated the incident overpressure and the reflected overpressure on a circular armor plate for C-4 and TNT types of explosives by utilizing the ALE formulation of the LS-Dyna code. Their model reflects a spherical mesh topology. Slavik [3] employed a mapping technique to couple the CONWEP blast loads to the ALE domain that utilizes various mesh resolutions. Kwasniewski et al. [4] examined the effects of different mesh resolutions and standoff distances on the reflected overpressure, and compared results with the available experiments. They concluded that the ALE simulation is highly sensitive to the mesh resolution used. Kilic [5] investigated the effect of the mesh resolution on the blast simulations of protective perimeter walls using the ALE approach of the LS-Dyna code. Kilic and Smith [6,7] investigated the blast response of deformable and rigid structural walls for the protection of critical buildings by employing the CONWEP and the ALE approaches of the LS-Dyna code. They used the CONWEP approach to investigate the response of an individual blast wall, and used the ALE approach to simulate the shadowing effects of blast walls placed between the high explosive and the target structure for various standoff distances.

Erdik et al. [8] examined the effects of landmine explosives detonated in a steel pot placed under the V-shaped hull of an armored vehicle using the ALE formulation. They reported that the numerical results were in agreement with the experimental results.

Yin et al. [9] studied modeling the blast loads on building structures. They employed the ALE approach with the spherical mesh topology to model the high explosive and the surrounding air with one-to-one node transition at the boundary. They suggested that fine mesh resolutions should be used in the ALE method to provide accurate results for the incident and the reflected overpressures.

Zakrisson et al. [10] presented the numerical and experimental results of deformable steel plates subjected to explosives confined in a steel pot. They used the ALE formulation with different mesh resolutions and compared the results with the CONWEP approach. They concluded that the CONWEP approach was not suitable for representing the confinement effects of the explosive placed in the pot. They concluded that the CONWEP approach cannot accurately represent the blast wave formation for explosives with geometries other than the spherical or the hemispherical shapes.

The CONWEP approach requires the least amount of computational resources and includes the effects of the gaseous products of the explosion during the afterburning process after detonation. The CONWEP approach provides more accurate results in the near field range compared to the ALE technique; however, it cannot accurately represent the blast wave formation for non-spherical high explosive geometries. The ALE approach is computationally intensive but can sim- 
ulate the confinement effect for encased explosives, the effect of the shape of the high explosive, and can handle the blast wave reflections on complex target surfaces as well as blast shadowing effects when obstacles exist between the high explosive and the target structure. This paper aims to present the effects of mesh resolution as well as mesh topology on the blast simulation results using the ALE approach.

\section{Methodology}

There are two main approaches to simulate the dynamic pressure loading on target structures in the commercial finite element code LS-Dyna. The first method is based on applying a pre-defined pressure function directly on the nodes of the finite element model of the target structure. The predefined pressure-time history loading function is established upon the empirical equations obtained from experimental blast studies. Such empirical equations are available in the U.S. Army Manual TM 5-1300 [11]. A numerical standalone computer program, CONWEP was developed by the United States Army Corps of Engineers Protective Design Center that incorporates the empirical equations of the Army Design Manual TM 5-855-1 [12]. The same equations of the CONWEP program were also implemented in the LS-Dyna code for simulating blast loading of structures through the use of the * LOAD_BLAST keyword function $[13,14]$.

The other alternative method available in the LS-Dyna code is the ALE approach. The medium between the high explosive and the structure is explicitly modeled with a volumetric mesh using the Eulerian approach. The Eulerian mesh is divided into two parts; the fluid medium and the high explosive that share common nodes. The physical properties of air and the high explosive are assigned to the elements that represent the fluid medium and the explosive device, respectively. When the detonation process takes place, the blast wave travels at the user-input detonation velocity inside the mesh domain of the explosive material. The blast wave is induced in the fluid medium when the wave reaches the shared nodes of the high explosive-fluid boundary. The blast wave then travels in the fluid domain. The interaction between the blast wave and the target structure is provided using the *CONSTRAINED_LAGR_IN_SOLID keyword function in the LS-Dyna code. The fluid-structure interaction method is based on a penalty formulation [15]. The target structure is modeled by a Lagrangian mesh that does not share nodes with the Eulerian fluid domain. This allows the analyst to insert any arbitrary Lagrangian mesh of the target structure inside the volumetric Eulerian fluid mesh.

The CONWEP method is used for the calibration of the blast load parameters of the ALE approach for the TNT type of high explosive material investigated in this study. When explosives of other types are employed, the approach relies on

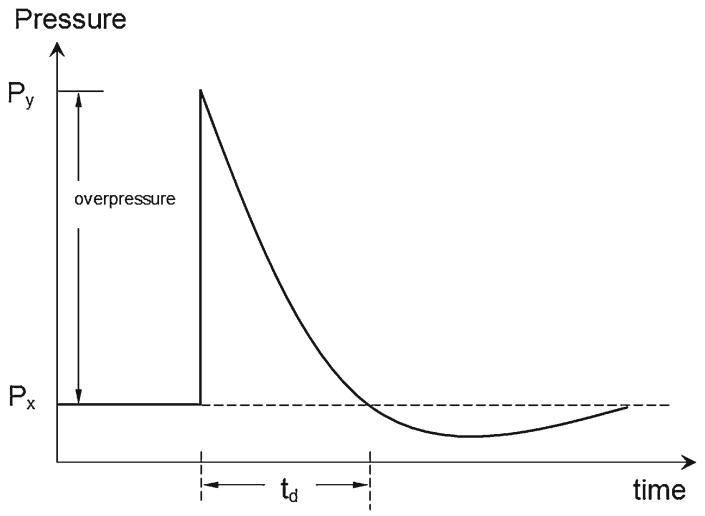

Fig. 1 Blast wave pressure-time profile

an equivalent TNT mass. However, the TNT equivalence may depend on a number of parameters such as charge size, scaled distance, detonation speed, Chapman-Jouguet pressure; and for a given explosive the pressure and the impulse calculations may require different TNT equivalence values [16-18]. Therefore, it is difficult to handle non-TNT types of explosives with the CONWEP approach. In contrast, the ALE method allows the adjustment of material properties and the equation of state parameters for various non-TNT explosives. These parameters are given in the study of Dobratz and Crawford [19].

The incident and reflected blast overpressures of the CONWEP loading in the absence of an obstacle or reflections provide the more accurate solutions available for a TNT type of high explosive with a spherical geometry. The afterburning effects are included in the CONWEP calculations due to the fact that the CONWEP approach utilizes the experimental measurements through the use of empirical equations. A parametric validation study is often required in order to benchmark the ALE results with the CONWEP solutions. Although the ALE approach allows more complex scenarios to be modeled, it requires more inputs from the analyst such as fluid-structure interaction parameters, the physical properties of the high explosive and the fluid medium. The difference in the mesh resolutions of the Lagrangian and the Eulerian domains as well as the mesh topologies plays an important role on the fidelity of the results. In this study, the incident and reflected overpressure time histories of the ALE solutions are compared with the CONWEP results.

\subsection{Blast wave}

Typical blast wave pressure variation with respect to time has a steep rise followed by an exponential decay region as shown in Fig. 1. The rise of pressure to $P_{y}$ and its drop below the atmospheric pressure $P_{x}$ in a finite amount of time show that this behavior cannot be expressed as a simple logarithmic decay. The overpressure $p_{0}$ is defined as the difference 
Table 1 JWL equation of state parameters for TNT

\begin{tabular}{lllllllll}
\hline$\rho_{0}\left(\mathrm{~kg} / \mathrm{m}^{3}\right)$ & $D(\mathrm{~m} / \mathrm{s})$ & $P_{\mathrm{CJ}}(\mathrm{GPa})$ & $E_{0} / V(\mathrm{GPa})$ & $A(\mathrm{GPa})$ & $B(\mathrm{GPa})$ & $R_{1}$ & $R_{2}$ & $W$ \\
\hline 1630 & 6930 & 21.0 & 7.0 & 371.213 & 3.231 & 4.15 & 0.95 & 0.30 \\
\hline
\end{tabular}

between the pressures $P_{y}$ and $P_{x}$. An empirical correction factor is added to the logarithmic decay in order to ensure a quasi-exponential form as given in (1) [20]:

$p=p_{0}\left(1-\frac{t}{t_{d}}\right) e^{-\alpha\left(\frac{t}{t_{d}}\right)}$

The decay parameter is represented by $\alpha, t$ is time, $p$ is the instantaneous overpressure at time $t, p_{0}$ is the maximum overpressure, $t_{d}$ is the time duration, and $e$ is the base of natural logarithms. Kinney [20] provides the decay parameters for different $\frac{p}{p_{0}}$ and $\frac{t}{t_{d}}$ values. The relationship between blast impulse and decay parameter is then obtained by integration of (2):

$I=\int_{0}^{t_{d}} p d t=p_{0} t_{d}\left[\frac{1}{\alpha}-\frac{1}{\alpha^{2}}\left(1-e^{-\alpha}\right)\right]$.

\subsection{High explosive}

The NATO AEP-55 Vol. II [1] describes test conditions for NATO member countries to determine the protection level of logistic and light armored vehicles subjected to blast effects arising from grenade and landmine threats. The analysis procedure involves the detonation of the high explosive such as $\mathrm{C} 4$ or TNT at the center of the landmine by the use of applicable material models. TNT is used in this study as the high explosive material. The detonation velocity in the high explosive material determines the detonation time of a particle [20].

The evaluation of the explosive after ignition is described by the Jones-Wilkins-Lee (JWL) equation of state, defined with the keyword *EOS_JWL in the LS-Dyna code [15]. The JWL equation of state defines the pressure as a function of the relative volume $\mathrm{V}$ and initial internal energy per volume $E_{0}$ as given in (3) [15]:

$p=A\left[1-\frac{W}{R_{1} V}\right] e^{-R_{1} V}+B\left[1-\frac{W}{R_{2} V}\right] e^{-R_{2} V}+\frac{W E_{0}}{V}$

The input parameters are represented by $A, B, R_{1}, R_{2}, W$, and $E_{0} . A$ and $B$ have dimensions of pressure, while the dimensionless parameters are $R_{1}, R_{2}$, and $W . E_{0}$ represents the initial internal energy. The volumetric ratio is expressed by $V=\frac{v}{v_{0}}$, where $v_{o}$ is the initial volume. The exponential terms are the high-pressure small-volume terms, and usually the user chooses $\frac{R_{1}}{R_{2}} \cong 4$ to make the two terms important in different regions.
The parameters of the JWL equation of state for the TNT high explosive material provided by Dobratz and Crawford [19] are given in Table 1 . The detonation velocity is given by $D, P_{\mathrm{CJ}}$ is the Chapman-Jouguet detonation pressure, and $\rho_{0}$ is the initial density. The detonation process is modeled by the programmed burn approach available in the LS-Dyna code. Detonation is activated at the center of the high explosive at the beginning of the simulation. The reactive wave travels at a constant speed of $D$ inside the domain of the high explosive material [21]. After the completion of the detonation process the interaction with the air domain begins and the pressure wave is generated in the air medium.

When a high explosive has insufficient oxygen to react with the available carbon and hydrogen in its chemical formation, the explosive is classified as oxygen deficient. Oxygen deficient explosives tend to react with the ambient oxygen in the surrounding air medium after the formation of the shock wave by the ignition process. The afterburning reaction and the subsequent release of its chemical energy depend on reaching a threshold pressure and a threshold temperature as well as the availability of sufficient oxygen in the surrounding medium. Afterburn is a difficult phenomenon to model due to the fact that the initial detonation and the afterburn energy release on the timescale are in the order of microseconds and milliseconds, respectively [22]. The afterburning effect in a blast simulation is a time-delayed release of energy that corresponds to a secondary shock. The afterburning effect may increase the total impulse exerted on the target structure rather than increasing the peak overpressure $[23,24]$. Therefore, the exclusion of the afterburning effect may lead to an underestimation of the total impulse in the blast simulation. Some computer codes allow the modeling of the afterburn process using a modified form of the JWL equation of state. However, this option is not currently available in the LS-Dyna code. In contrast with the ALE technique, the CONWEP approach is based on experimental results. Therefore, the afterburning effect is included in the calculations carried out with the empirical CONWEP equations. The results using the ALE approach presented in this study for the flat plate simulations and the full-scale landmine blast simulation exclude the afterburning effect.

\subsection{Calculation domain}

The fluid-structure interaction (FSI) calculations are carried out in the Eulerian domain, which consists of the high explosive and the surrounding volume of air. The Lagrangian target structure is inserted into the Eulerian domain. However, the 
nodes of the Lagrangian mesh do not share common nodes with the Eulerian domain. The movement of the Lagrangian finite element mesh of the structure becomes independent of the movement of the material flow of fluids in the Eulerian domain when the ALE approach is used; thus, a greater flexibility is obtained in modeling the blast scenario. The Eulerian domain of the high explosive and the air volume is meshed with eight-node hexahedral elements.

The linear polynomial equation of state used for the air domain is given in (4) [21]. The coefficients $C_{0}$ through $C_{6}$ are the parameters supplied by the analyst. The variable $\mu$ depends on the volumetric ratio $V$ as given in (5):

$$
p=C_{0}+C_{1} \mu+C_{2} \mu^{2}+C_{3} \mu^{3}+\left(C_{4}+C_{5} \mu+C_{6} \mu^{2}\right) E
$$

$\mu=\frac{1}{V}-1$

In expanded elements the coefficients of $\mu^{2}$ are set to zero; thus, the coefficients $C_{2}$ and $C_{6}$ in (4) vanish. The linear polynomial equation of state may be used to model an ideal gas by setting the coefficients $C_{0}, C_{1}$, and $C_{3}$ to zero, and using the value given in (6) for the coefficients $C_{4}$ and $C_{5}$. The ratio of specific heats is expressed by $\gamma$ with a value of 1.4 used for the air medium. Inserting the values of the parameters $C_{0}$ through $C_{6}$ into (4), the pressure is finally obtained in (7). The internal energy per unit volume is given by $E$. The initial and current densities of air are represented by $\rho_{0}$ and $\rho$, respectively.

$C_{4}=C_{5}=\gamma-1$

$p=(\gamma-1) \frac{\rho}{\rho_{0}} E$

\section{Rigid reflecting surface study}

Element size is a crucial factor that affects the results of blast simulations. Therefore, mesh resolution and mesh topology should be given special care in constructing the finite element model. However, factors such as time step marching algorithm, fluid-structure coupling methods, and finite element code capabilities may limit the accuracy of the blast simulations even when fine mesh resolutions are used.

The CONWEP calculations are well documented in the available literature [11-13]. The empirical equations employed by the CONWEP program are based on the measurements obtained from various blast experiments. Therefore, it is essential to compare the CONWEP calculations with the ALE simulation results in order to validate the numerical studies. The peak incident overpressure and the peak reflected overpressure of the ALE simulations converge to the CONWEP calculations for fine mesh resolutions.
However, ALE simulations with coarse mesh resolutions significantly underestimate the incident overpressure and the reflected overpressure of the CONWEP results. The comparisons of the incident impulse and the reflected impulse show a similar trend.

The blast response of a rigid reflecting surface placed opposite to an explosive charge is investigated in order to assess the influence of mesh resolution and mesh topology effects on the ALE simulations. The parametric study utilizes Cartesian and spherical topologies with varying mesh resolutions. The purpose of the parametric study is to build a basis for blast simulations that involve complex geometric configurations.

\subsection{Mesh topologies: cartesian and spherical meshes}

The parametric ALE studies are classified in accordance with the ratio of the element sizes of the Eulerian and the Lagrangian domains at the region of interest. Since the fluidstructure interaction plays a major role on the fidelity of the blast simulations, the region of interest is at the coupling interface of the Eulerian and the Lagrangian domains.

For instance, the C6 simulation refers to the Cartesian type Eulerian mesh with the $\mathrm{C}$ designation and it consists of 6 Eulerian elements interfacing with a single Lagrangian shell element at the coupling region. The Cartesian meshes used in this study have a cubical mesh topology and the mesh resolutions are $\mathrm{C} 1, \mathrm{C} 2, \mathrm{C} 4, \mathrm{C} 6, \mathrm{C} 8$, and $\mathrm{C} 10$.

Similarly, the S6 simulation utilizes the spherical topology (S designation) for the Eulerian domain with 6 elements interfacing with a single Lagrangian shell element at the coupling region. It should be noted that the size of the Eulerian elements increases in the radial direction for the spherical mesh topology. The spherical mesh resolutions used in this study are S1, S2, S4, S6, S8, and S10. The spherical mesh domain is in the shape of a complete sphere. Table 2 shows the number of elements and the number of nodes for the rigid reflecting surface parametric study.

Figure $2 \mathrm{a}$ and $\mathrm{b}$ provides the setup of the Cartesian and spherical blast simulations. The target Lagrangian plate structure is placed at a distance of $500 \mathrm{~mm}$ to the high explosive charge. The airburst of a TNT charge of $1 \mathrm{~kg}$ is investigated for a scaled distance $Z$ value of $0.5 \mathrm{~m} / \mathrm{kg}^{\frac{1}{3}}$.

The model consists of the high explosive, the surrounding volume of air, and the rigid reflecting surface. The Cartesian topology of the high explosive consists of cube elements with an edge length of $8.5 \mathrm{~mm}$. For the spherical topology, the high explosive geometry is a sphere with a radius of $5.27 \mathrm{~mm}$. The volume of the mesh domain of the high explosive material is $614 \mathrm{~mm}^{3}$ in both topologies in order to obtain a charge mass of $1 \mathrm{~kg}$. The high explosive charge and the surrounding air are modeled with hexahedral Eulerian elements, whereas 
Table 2 Parametric study mesh details for the Cartesian and spherical topologies

\begin{tabular}{lll}
\hline Model & No. of elements $\left(\times 10^{6}\right)$ & No. of nodes $\left(\times 10^{6}\right)$ \\
\hline C1 & 0.005 & 0.006 \\
C2 & 0.039 & 0.043 \\
C4 & 0.314 & 0.329 \\
C6 & 1.061 & 1.093 \\
C8 & 2.515 & 2.571 \\
C10 & 4.913 & 5.000 \\
S1 & 0.007 & 0.007 \\
S2 & 0.056 & 0.057 \\
S4 & 0.451 & 0.454 \\
S6 & 1.521 & 1.528 \\
S8 & 3.604 & 3.617 \\
S10 & 7.040 & 7.060 \\
\hline
\end{tabular}

the target plate structure is modeled with a single four-node Lagrangian shell element.

Figure 3 illustrates the finite element meshes for the $\mathrm{C} 1$ and the S1 simulations, respectively. Close-up views of the mesh region around the high explosive domains are also shown in Fig. 3. The size of the surrounding air elements is constant in the entire Eulerian mesh for the $\mathrm{C} 1$ Cartesian mesh simulation. However, the size of the surrounding air elements increases in the radial direction away from the high explosive device for the S1 spherical mesh simulation. The size of the surrounding air and the high explosive elements are the same at the coupling boundary for both simulations.

The detonation is initiated at the centroid of high explosive domain in the Cartesian and the spherical mesh topologies using the programmed burn option in the LS-Dyna code. As the $\mathrm{C} 1$ simulation has a single hexahedral element used to model the high explosive, the detonation takes place at the centroid of the element shown in the close-up view in Fig. 3(a). The S1 simulation has multiple hexahedral elements to model the spherical shape of the high explosive domain as illustrated in Fig. 3(b).

\subsection{Incident and reflected overpressures}

One of the crucial steps in measuring the reflected overpressure is to choose an appropriate number of integration points in the quadrature rule used in the ALE fluid-structure interaction algorithm of the LS-Dyna code. The NQUAD parameter refers to the number of quadrature points for the fluidstructure interaction. In order to couple a single Lagrangian element to multiple Eulerian elements, the NQUAD parameter defines a grid of $n$-by- $n$ integration points at the fluidstructure coupling surface. If the Lagrangian and Eulerian element edge lengths are similar, a value of 2 is sufficient for

Fig. 2 Schematic drawing

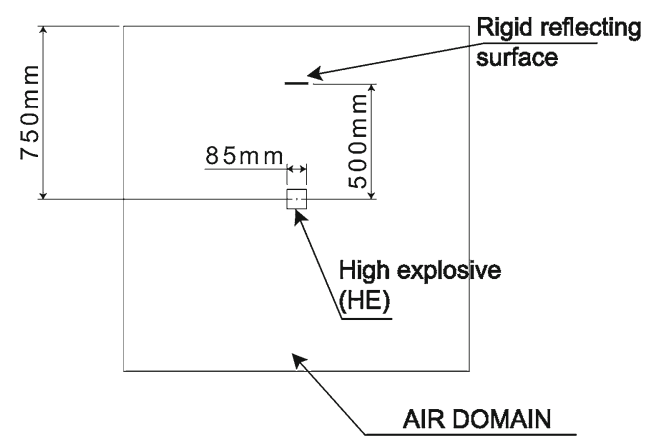

(a) Cartesian mesh domain

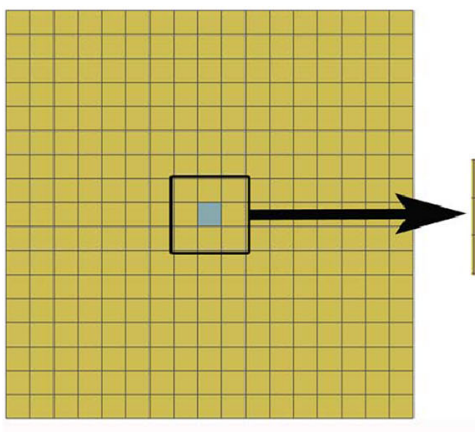

(a)

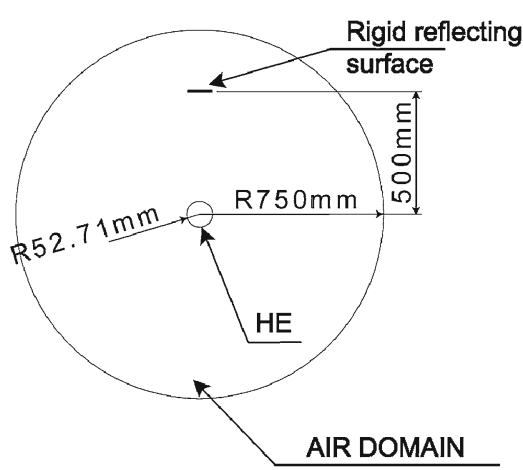

(b) Eulerian mesh domain
Fig. 3 Mesh discretization of (a) Cartesian (C1) and (b) spherical (S1) Eulerian mesh topologies consisting of surrounding air and high explosive
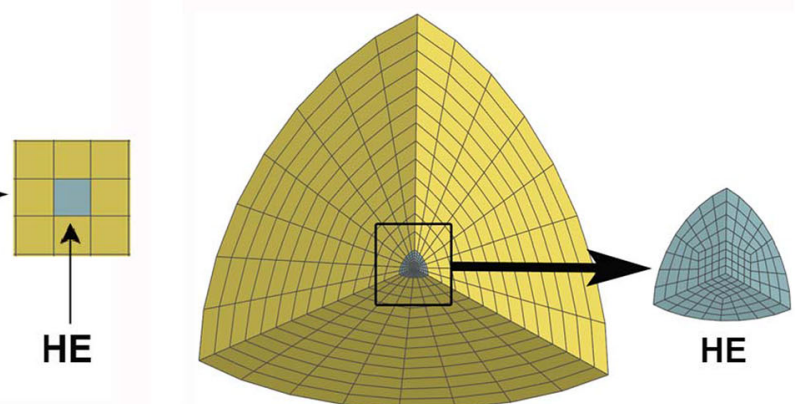

(b) 
Table 3 Spherical free-air burst CONWEP calculation of a $1 \mathrm{~kg}$ TNT charge

\begin{tabular}{ll}
\hline Equivalent weight of TNT $(\mathrm{kg})$ & 1.00 \\
Range to target $(\mathrm{m})$ & 0.50 \\
Time of Arrival $(\mathrm{msec})$ & 0.1541 \\
Peak incident overpressure $(\mathrm{kPa})$ & 3885.0 \\
Peak normally reflected overpressure $(\mathrm{kPa})$ & 30360.0 \\
Positive phase duration $(\mathrm{msec})$ & 0.3196 \\
Incident impulse $(\mathrm{kPa}-\mathrm{msec})$ & 141.3 \\
Reflected impulse $(\mathrm{kPa}-\mathrm{msec})$ & 1454.0 \\
Shock front velocity $(\mathrm{m} / \mathrm{sec})$ & 1953.0 \\
\hline
\end{tabular}

the NQUAD parameter. For the combination of fine mesh resolution in the Eulerian domain and coarse mesh resolution in the Lagrangian domain, the value of the NQUAD parameter should be increased.

The body of armored vehicles is modeled with Lagrangian shell elements. Computational fluid dynamics equations generally require fine mesh resolutions in the Eulerian domain. It is common practice that the blast simulation of an armored vehicle involves coarse and fine mesh resolutions in the Lagrangian domain and the Eulerian domain, respectively. In our study, we found out that the optimum value of the NQUAD parameter is the number of Eulerian elements that share a common interface edge with a Lagrangian element. For example, a single Lagrangian element that is coupled with six Eulerian elements on the interface edge should utilize a value of 6 for the NQUAD parameter as in the case of the C6 Cartesian mesh and the $\mathrm{S} 6$ spherical mesh simulations.

Table 3 shows the summary of the CONWEP calculation results for the spherical airburst of a $1 \mathrm{~kg}$ TNT charge with a standoff distance of $0.5 \mathrm{~m}$. For the selected parameters, the peak normally reflected overpressure and the peak reflected impulse are an order of magnitude higher than the peak incident overpressure and the peak incident impulse, respectively.

\subsubsection{Comparison of incident overpressure with the CONWEP calculations}

Figure 4 presents the variation of the incident overpressure over time for the $\mathrm{C} 1-\mathrm{C} 10$ Cartesian simulations. The peak overpressure is significantly underestimated for the $\mathrm{C} 1, \mathrm{C} 2$, and $\mathrm{C} 4$ mesh resolutions when compared with the $\mathrm{CON}$ WEP calculation. The arrival times of the shock front for the $\mathrm{C} 1, \mathrm{C} 2$, and $\mathrm{C} 4$ simulations do not match the CONWEP calculations. For the higher mesh resolutions, the deviation percentage for the peak overpressure becomes 42, 21, and 2 for the $\mathrm{C} 6, \mathrm{C} 8$, and $\mathrm{C} 10$ simulations, respectively. The arrival time of the shock front also improves for the higher mesh resolutions of $\mathrm{C} 6, \mathrm{C} 8$, and $\mathrm{C} 10$.

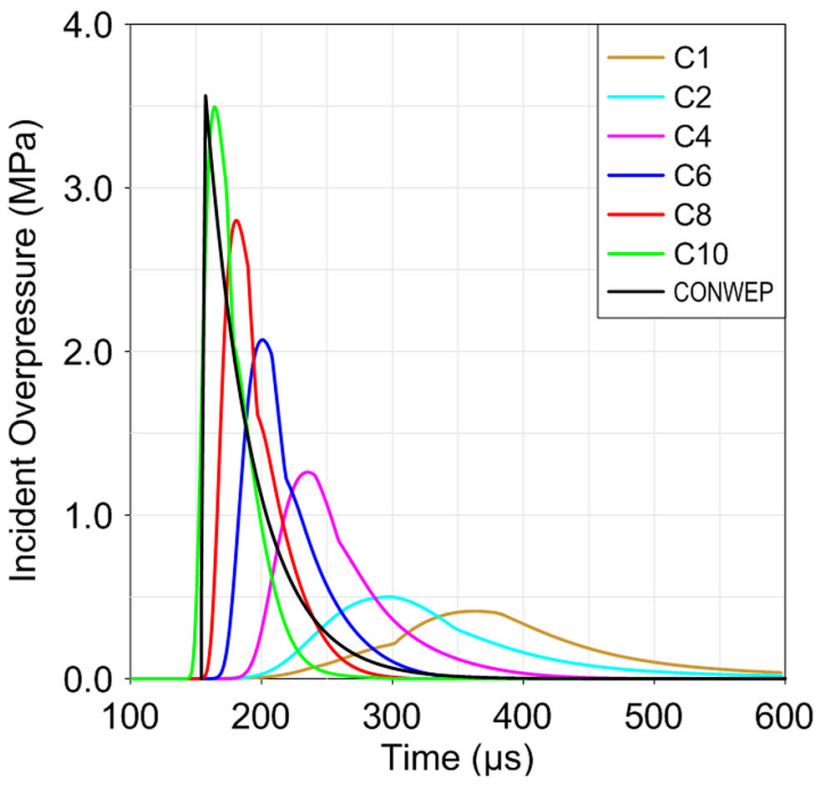

Fig. 4 Incident overpressure for the Cartesian topology

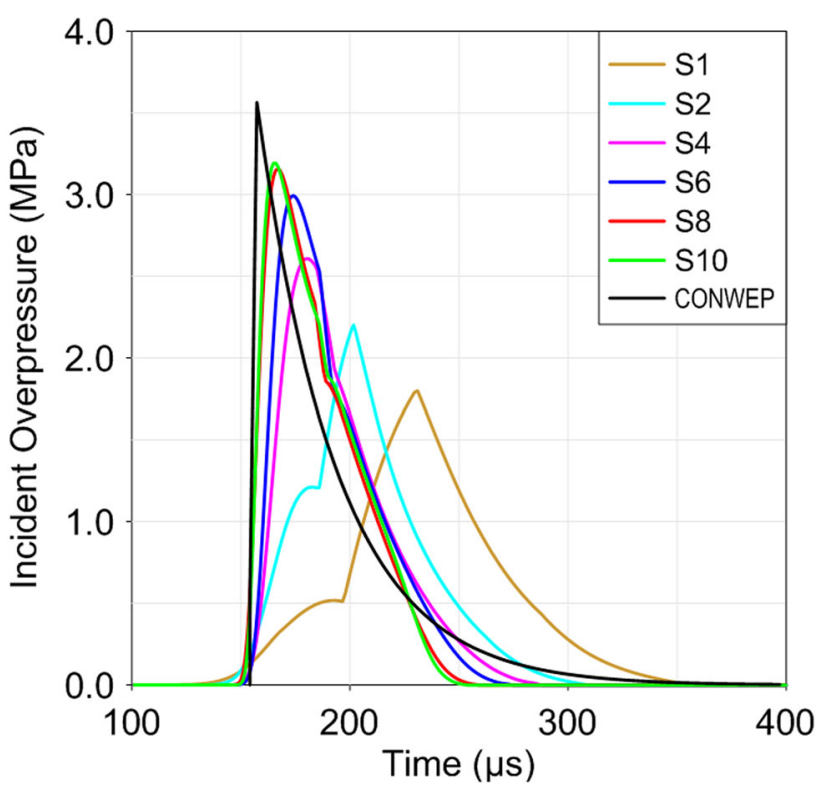

Fig. 5 Incident overpressure for the spherical topology

Figure 5 illustrates the variation of the incident overpressure over time for the $\mathrm{S} 1-\mathrm{S} 10$ spherical simulations. The arrival time of the shock front is in close proximity with the CONWEP calculations for all mesh resolutions when compared with the Cartesian simulations. The deviation percentage of the peak overpressure of the S6, S8, and S10 simulations is 16,11 , and 10 , respectively.

Table 4 provides the total number of elements, the peak incident overpressures, and the deviation percentages compared with the CONWEP calculations. 
Table 4 Number of elements, peak incident overpressure, and deviation percentages compared with the CONWEP calculations

\begin{tabular}{llll}
\hline Model & $\begin{array}{l}\text { Number of } \\
\text { elements }\left(\times 10^{6}\right)\end{array}$ & $\begin{array}{l}\text { Peak incident } \\
\text { overpressure } \\
\left(\times 10^{-5} \mathrm{Mbar}\right)\end{array}$ & $\begin{array}{l}\text { Deviation } \\
\text { percentage }(\%)\end{array}$ \\
\hline C1 & 0.005 & 0.41 & 88 \\
C2 & 0.039 & 0.50 & 86 \\
C4 & 0.314 & 1.26 & 65 \\
C6 & 1.061 & 2.07 & 42 \\
C8 & 2.515 & 2.80 & 21 \\
C10 & 4.913 & 3.49 & 2 \\
S1 & 0.007 & 1.80 & 50 \\
S2 & 0.056 & 2.20 & 38 \\
S4 & 0.451 & 2.61 & 27 \\
S6 & 1.521 & 2.99 & 16 \\
S8 & 3.604 & 3.16 & 11 \\
S10 & 7.040 & 3.19 & 10 \\
\hline
\end{tabular}

\subsubsection{Comparison of reflected overpressure with the CONWEP calculations}

The purpose of realistic blast simulations is to investigate the response of a structural entity when subjected to blast loads. Reflection of shock waves from structural surfaces requires the use of complex FSI algorithms. Therefore, simulations involving the interaction of blast waves with structures are more challenging than simulating the free expansion of blast waves in air [20].

Figure 6 illustrates the variation of the reflected overpressure over time for the C1-C10 Cartesian simulations. Similar to the observations obtained in the peak incident overpressures, the peak reflected overpressure is underestimated for the $\mathrm{C} 1, \mathrm{C} 2$, and $\mathrm{C} 4$ mesh resolutions. There is a significant gap between the arrival times of the shock front for the $\mathrm{C} 1, \mathrm{C} 2$, and $\mathrm{C} 4$ simulations and the CONWEP calculations. The deviation percentage for the peak overpressure gradually improves to 48, 33, and 19 for the C6, C8, and C10 simulations, respectively. An improvement of the arrival time of the shock front is also observed for the $\mathrm{C} 6, \mathrm{C} 8$, and $\mathrm{C} 10$ simulations. Table 4 shows $2 \%$ deviation for the peak incident overpressure for the $\mathrm{C} 10$ simulation. However, the deviation for the peak normally reflected overpressure is $19 \%$ for the C10 simulation in Table 5. The contrast between the two deviation margins illustrates the difficulty of simulating reflected shock waves.

Figure 7 shows the variation of the reflected overpressure over time for the S1-S10 spherical simulations. The arrival times of the shock front are generally in agreement with the CONWEP calculations with the exception of the S1 simulation. The deviation percentages of the peak reflected overpressure are in the high ranges for the S1, S2, and S4

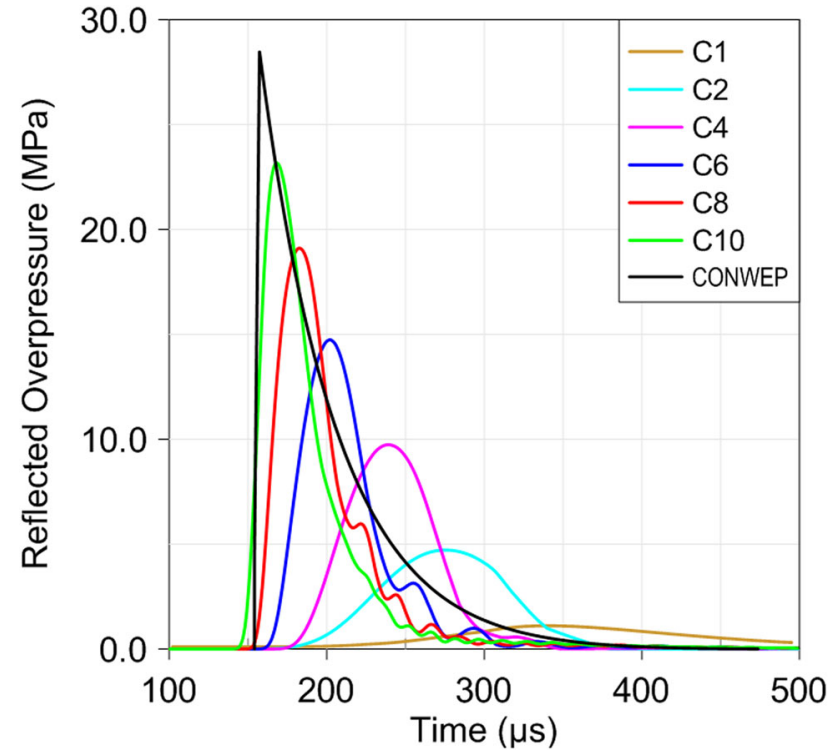

Fig. 6 Reflected overpressure for the Cartesian topology

simulations with coarse mesh resolutions. The deviation percentage is reduced to 24,17 , and 9 for the S6, S8, and S10 simulations, respectively. The deviation percentage is halved in the spherical topology when compared with the Cartesian topology deviation percentages of 48, 33, and 19 of the C6, $\mathrm{C} 8$, and $\mathrm{C} 10$ simulations, respectively.

In order to compare the blast wave propagation at similar intervals for the C6 Cartesian and the S6 spherical simulations, contour plots of pressure are needed. Figures 8, 9, and 10 show the pressure contour plots of the C6 case for the time instances of 49.9, 99.9, and $209.7 \mu \mathrm{sec}$, respectively. The square shape of the high explosive does not yield a perfectly spherical blast wave profile at any time instance. However, the CONWEP approach for the flat plate assumes that the high explosive charge has a spherical shape. The discrepancies between the C6 ALE simulation results and the CONWEP calculations are expected for this reason. The flow of the mesh lines of the C6 Cartesian simulation is not always perpendicular to the propagation direction of the blast wave.

Figures 11, 12, and 13 show the contour plots of the pressure of the S6 spherical simulation for the time instances of 50.0, 110.0, and $209.9 \mu \mathrm{sec}$, respectively. Since the high explosive charge has a spherical shape, the blast wave propagation is spherical as shown by the contour plots of pressure. Furthermore, the flow of the mesh lines is perpendicular to the propagation direction of the blast wave, creating an optimum situation for the advection algorithm of the ALE technique.

Table 5 shows the total number of elements, the ratio of the Eulerian elements interfacing with a single Lagrangian shell element, the value used for the NQUAD parameter in the LS-Dyna code, the peak reflected overpressures, and the deviation percentages compared with the CONWEP 
Table 5 Number of elements, Euler/Lagrange element ratio $(\mathrm{E} / \mathrm{L})$, value of the NQUAD parameter, peak reflected overpressures, and deviation percentages compared with the CONWEP calculations

\begin{tabular}{|c|c|c|c|c|c|c|}
\hline Model & $\begin{array}{l}\text { Number of } \\
\text { elements }\left(\times 10^{6}\right)\end{array}$ & $\begin{array}{l}\text { Ratio of Eulerian } \\
\text { elements to } \\
\text { Lagrangian } \\
\text { elements }\end{array}$ & NQUAD & $\begin{array}{l}\text { Peak reflected } \\
\text { overpressure } \\
\left(\times 10^{-4} \mathrm{Mbar}\right)\end{array}$ & Deviation (\%) & $\begin{array}{l}\text { Simulation } \\
\text { Run-time (total } \\
\text { CPU-seconds) }\end{array}$ \\
\hline $\mathrm{C} 1$ & 0.005 & 1 & 1 & 0.11 & 96 & 3 \\
\hline $\mathrm{C} 2$ & 0.039 & 2 & 2 & 0.47 & 83 & 13 \\
\hline $\mathrm{C} 4$ & 0.314 & 4 & 4 & 0.97 & 66 & 40 \\
\hline C6 & 1.061 & 6 & 6 & 1.47 & 48 & 128 \\
\hline $\mathrm{C} 8$ & 2.515 & 8 & 8 & 1.91 & 33 & 388 \\
\hline $\mathrm{C} 10$ & 4.913 & 10 & 10 & 2.32 & 19 & 909 \\
\hline S1 & 0.007 & 1 & 1 & 1.08 & 62 & 6 \\
\hline $\mathrm{S} 2$ & 0.056 & 2 & 2 & 1.70 & 40 & 20 \\
\hline S4 & 0.451 & 4 & 4 & 1.90 & 33 & 220 \\
\hline S6 & 1.521 & 6 & 6 & 2.18 & 24 & 3455 \\
\hline S8 & 3.604 & 8 & 8 & 2.35 & 17 & 3823 \\
\hline S10 & 7.040 & 10 & 10 & 2.57 & 9 & 7614 \\
\hline
\end{tabular}

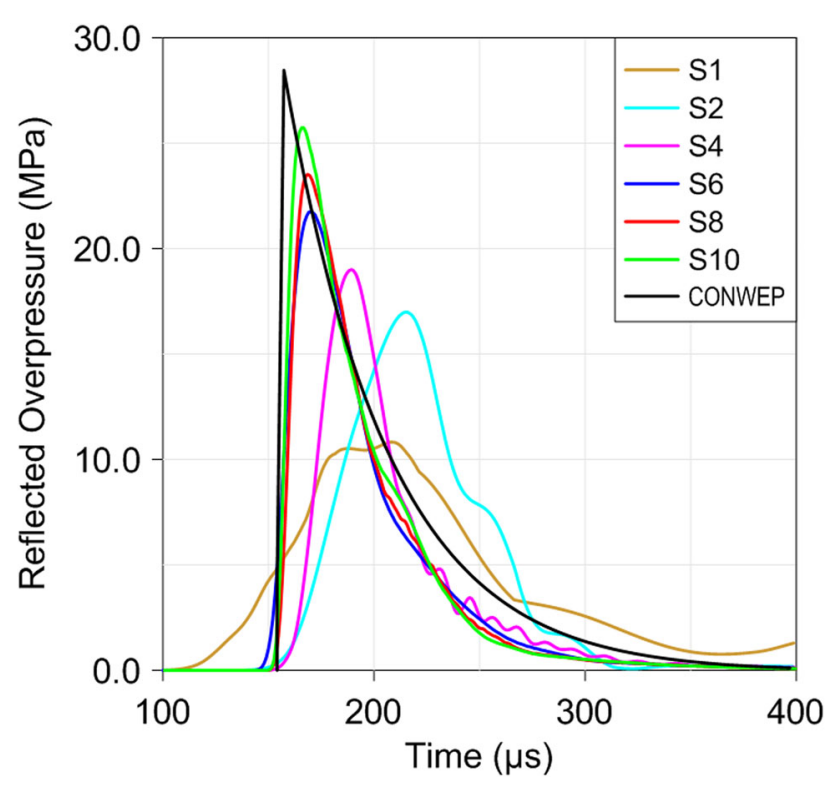

Fig. 7 Reflected overpressure for the spherical topology
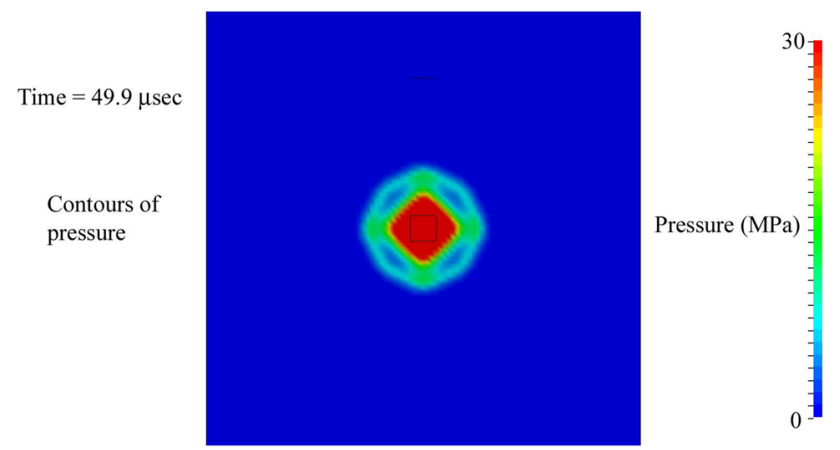

Fig. 8 Contours plots of pressure at $t=49.9 \mu \mathrm{sec}$ for the C6 simulation

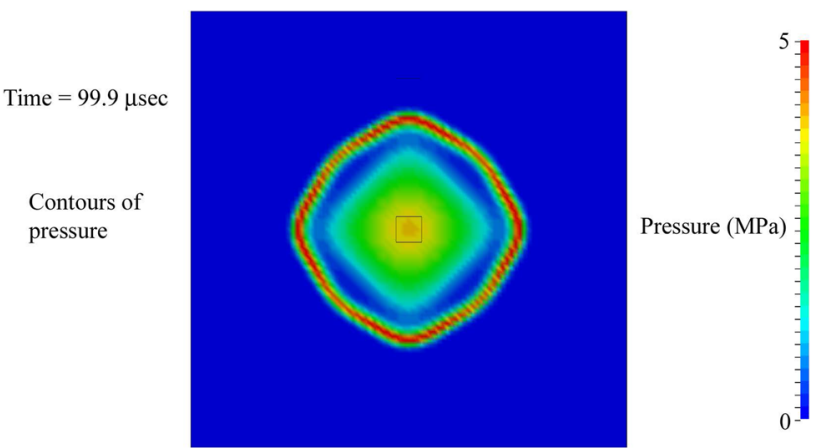

Fig. 9 Contours plots of pressure at $t=99.9 \mu \mathrm{sec}$ for the C6 simulation

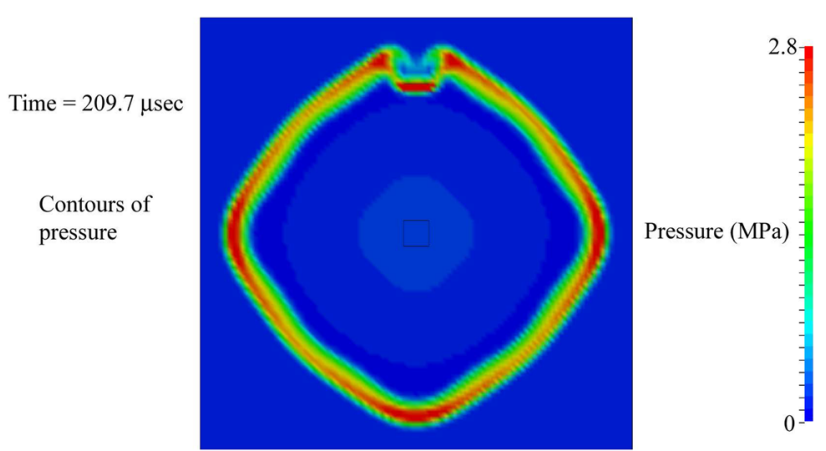

Fig. 10 Contours plots of pressure at $t=209.7 \mu \mathrm{sec}$ for the C6 simulation

calculations. The simulation run-times are also provided in Table 5. The simulations were carried out on the Windows HPC Server 2008 computing cluster with 36 processors and 216GB of RAM memory at the OTOKAR Otomotiv ve Savunma Sanayi A.S. Corporation. 


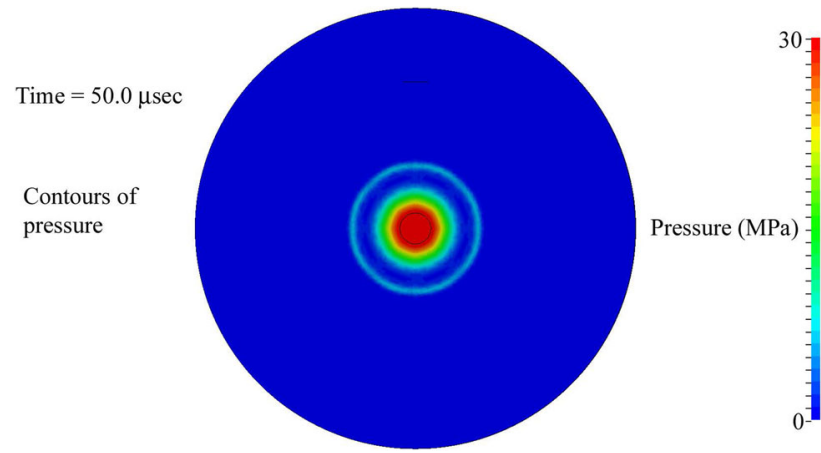

Fig. 11 Contours plots of pressure at $t=50.0 \mu \mathrm{sec}$ for the S6 simulation

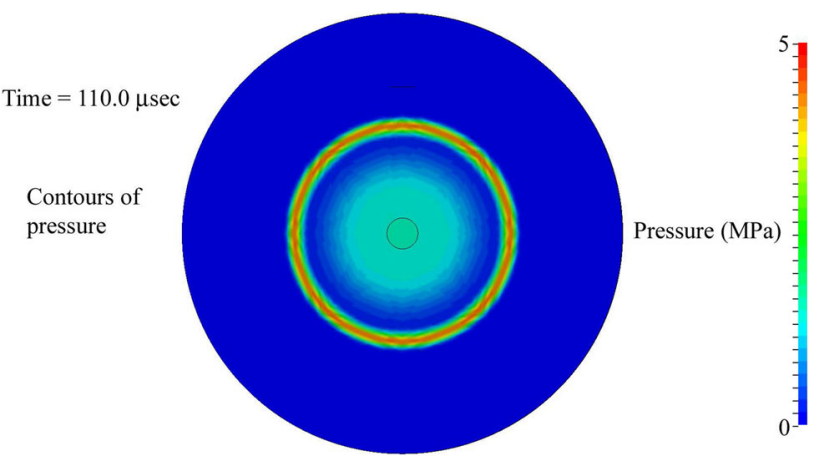

Fig. 12 Contours plots of pressure at $t=110.0 \mu \mathrm{sec}$ for the S6 simulation

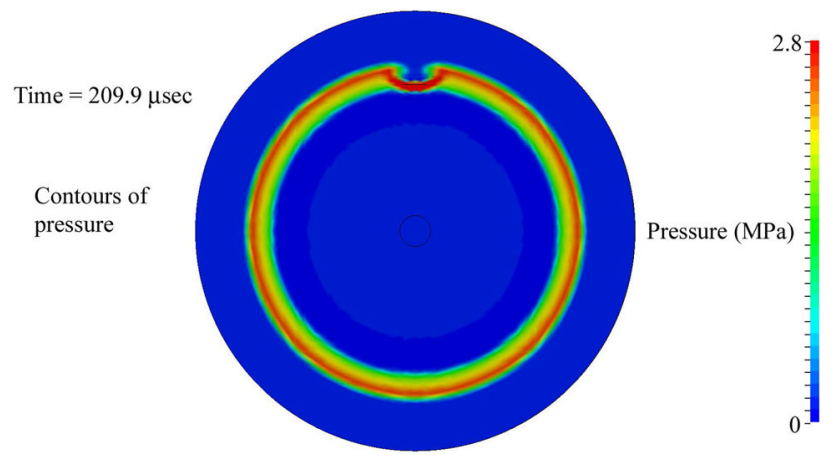

Fig. 13 Contours plots of pressure at $t=209.9 \mu \mathrm{sec}$ for the S6 simulation

\subsection{Incident and reflected impulses}

The blast response of a structure is sensitive to the reflected overpressures applied on the surfaces exposed to the shock wave. However, the peak reflected overpressure is not sufficient to express the dynamic response of the structure. The duration of the overpressure as well as its time variation plays important roles in determining the structural response [25]. The integration of the reflected overpressure variation with respect to time yields the reflected impulse. In blast scenarios that involve close proximity to the high explosive the struc-

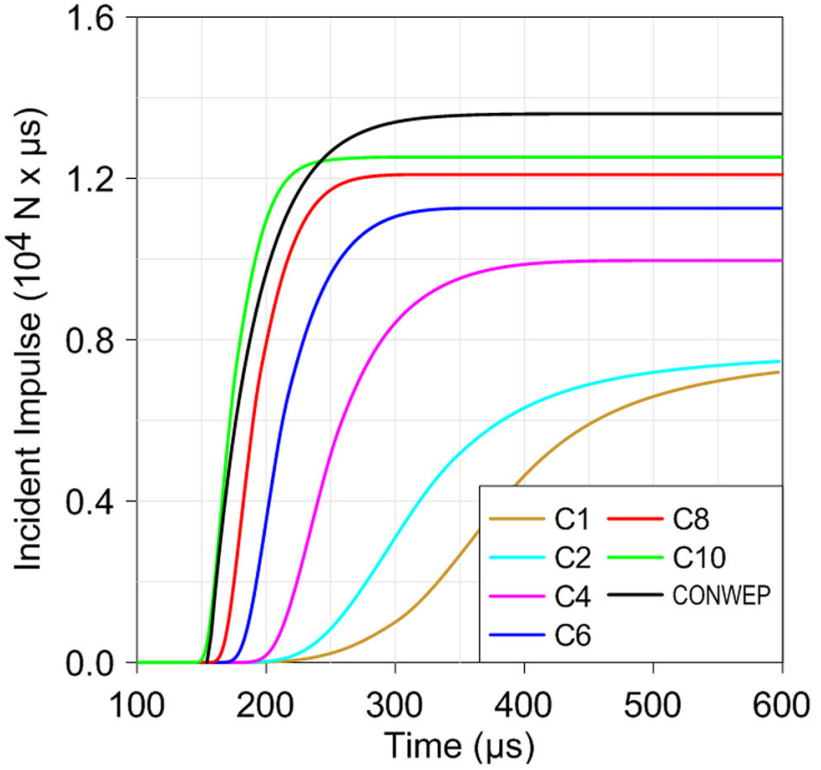

Fig. 14 Incident impulse for the Cartesian topology

tural response is extremely sensitive to the impulse levels. Therefore, this section provides the results for the incident impulse and the reflected impulse for the rigid reflecting surface parametric study.

\subsubsection{Comparison of incident impulse with the CONWEP calculations}

Figure 14 shows the time variation of the cumulative incident impulse for the C1-C10 Cartesian simulations. Only the C6, $\mathrm{C} 8$, and $\mathrm{C} 10$ simulations fall in the proximity of the CONWEP calculations with deviation margins of 17,11 , and 8 , respectively. Both the shock wave arrival time and the peak incident overpressure of the C6 simulation show significant variation when compared with the CONWEP calculations. The peak incident overpressure is underestimated for the $\mathrm{C} 8$ simulation. The positive phase duration of the $\mathrm{C} 10$ simulation is shorter when compared with the CONWEP calculations. Mesh resolution improves the incident impulse in the simulations.

Figure 15 shows the time variation of the cumulative incident impulse for the spherical mesh topology. The S6 simulation underestimates the CONWEP calculation with a deviation margin of $1 \%$. The S8 and S10 simulations overestimate the CONWEP calculation by $1 \%$ and $2 \%$, respectively. For lower mesh resolutions, there is a significant gap between the simulation results and the CONWEP calculation.

Table 6 provides the cumulative incident impulse and the deviation percentages compared with the CONWEP calculations for the Cartesian and spherical topologies. The spherical topology provides an advantage over the Cartesian topology for all mesh resolutions in the parametric study. 


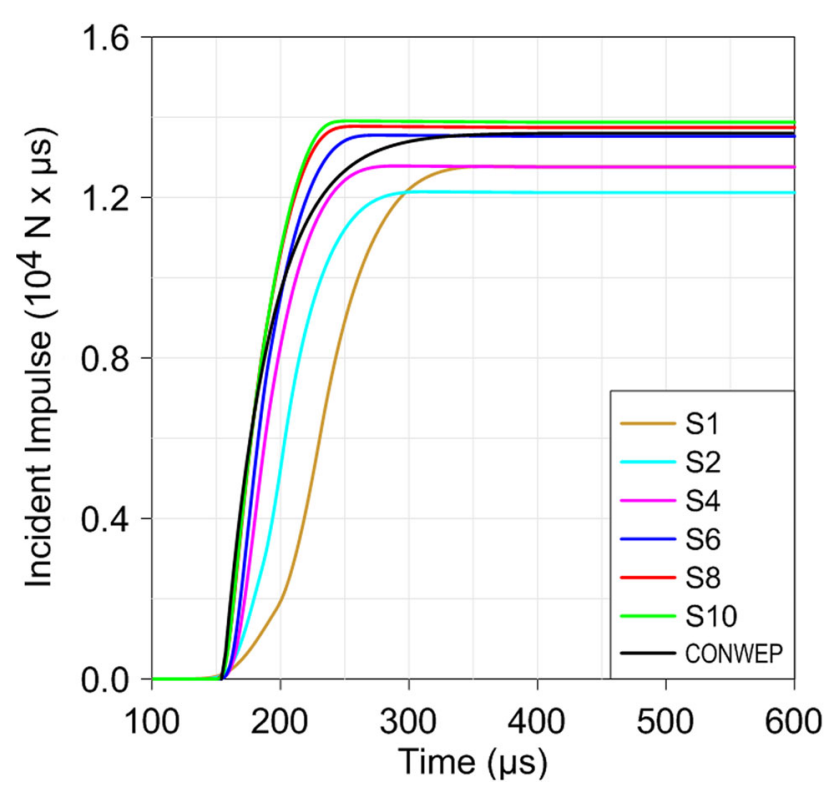

Fig. 15 Incident impulse for the spherical topology

\subsubsection{Comparison of reflected impulse with the CONWEP calculations}

Figure 16 shows the comparison of the reflected impulse time variation for the Cartesian mesh topology. The maximum and the minimum deviation margins are 90 and $25 \%$ for the $\mathrm{C} 1$ and $\mathrm{C} 10$ simulations, respectively. It is clear from the results of the parametric study that the Cartesian topology has a significant disadvantage for simulating the reflected impulse.

Figure 17 presents the time variation of the cumulative reflected impulse for the spherical mesh topology. The deviation margins of the S6, S8, and S10 simulations are 19, 20, and $15 \%$, respectively. Although the coarse resolution mesh topologies of S1 and S2 yield low deviations for the reflected

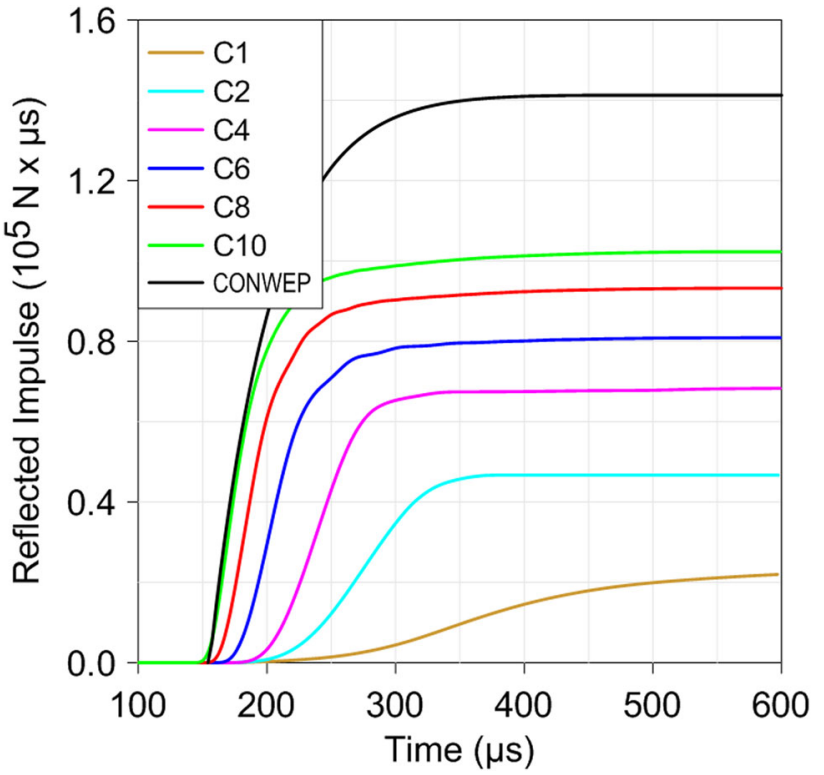

Fig. 16 Reflected impulse for the Cartesian topology

impulse, they have high deviations in terms of the reflected overpressure, and their pressure-time profiles do not reflect the typical blast wave profile as illustrated in Fig. 1.

Table 7 shows the cumulative reflected impulse for the Cartesian and spherical topologies and the deviation percentages compared with the CONWEP calculations. The Cartesian mesh topology shows a consistent trend of decreasing deviation percentages for the reflected impulse and overpressure as the mesh density increases. For a given mesh resolution with matching pairs such as $\mathrm{C} 4$ and $\mathrm{S} 4$, the spherical mesh topology provides better results for both quantities for the entire parametric study.

The total impulse achieved using the highest mesh resolutions in the C10 Cartesian and the S10 spherical simulations
Table 6 Cumulative incident impulse values of the simulations and the deviation percentages compared with the CONWEP calculations

\begin{tabular}{llll}
\hline Model & $\begin{array}{l}\text { Cumulative incident } \\
\text { impulse }\left(\times 10^{4} \mathrm{~N} . \mu \mathrm{sec}\right)\end{array}$ & $\begin{array}{l}\text { Deviation percentage } \\
\text { for incident impulse }(\%)\end{array}$ & $\begin{array}{l}\text { Deviation percentage } \\
\text { for incident overpressure } \\
(\%)\end{array}$ \\
\hline C1 & 0.72 & 47 & 88 \\
C2 & 0.75 & 45 & 86 \\
C4 & 1.00 & 27 & 65 \\
C6 & 1.13 & 17 & 42 \\
C8 & 1.21 & 11 & 21 \\
C10 & 1.25 & 8 & 2 \\
S1 & 1.28 & 6 & 50 \\
S2 & 1.21 & 11 & 38 \\
S4 & 1.28 & 6 & 27 \\
S6 & 1.35 & 1 & 16 \\
S8 & 1.38 & 1 & 11 \\
S10 & 1.39 & 2 & 10 \\
\hline
\end{tabular}




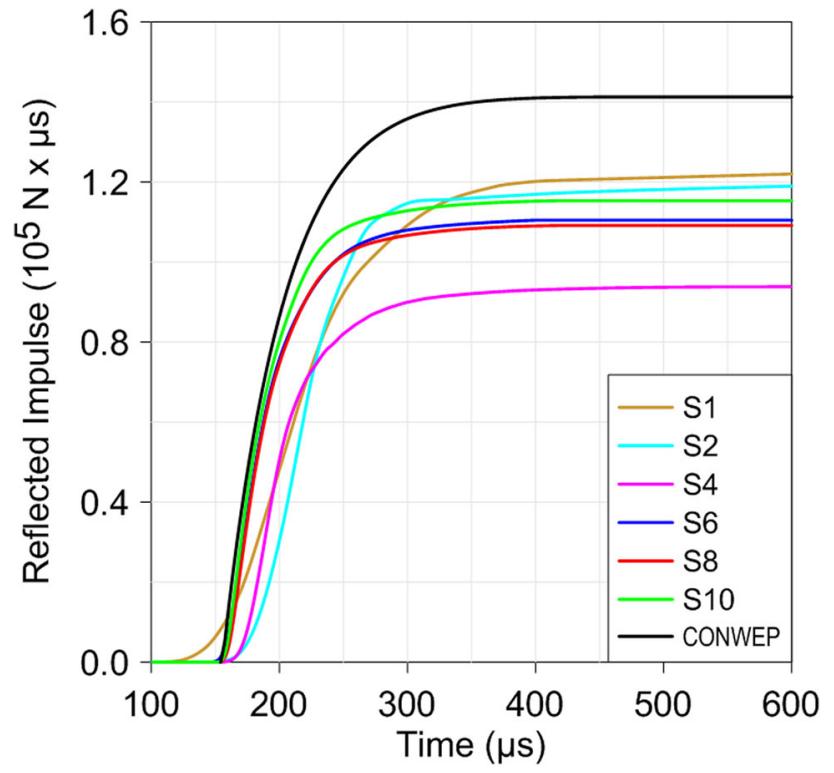

Fig. 17 Reflected impulse for the spherical topology

Table 7 Cumulative reflected impulse values of the simulations and the deviation percentages compared with the CONWEP calculations

\begin{tabular}{|c|c|c|c|}
\hline Model & $\begin{array}{l}\text { Peak reflected } \\
\text { impulse }\left(\times 10^{5}\right. \\
\text { N. } \mu \text { sec })\end{array}$ & $\begin{array}{l}\text { Deviation percent } \\
\text { age reflected for } \\
\text { impulse }(\%)\end{array}$ & $\begin{array}{l}\text { Deviation percent } \\
\text { age for reflected } \\
\text { overpressure }(\%)\end{array}$ \\
\hline $\mathrm{C} 1$ & 0.14 & 90 & 96 \\
\hline $\mathrm{C} 2$ & 0.47 & 65 & 83 \\
\hline $\mathrm{C} 4$ & 0.68 & 50 & 66 \\
\hline C6 & 0.80 & 41 & 48 \\
\hline C8 & 0.92 & 32 & 33 \\
\hline $\mathrm{C} 10$ & 1.01 & 26 & 19 \\
\hline $\mathrm{S} 1$ & 1.22 & 10 & 62 \\
\hline $\mathrm{S} 2$ & 1.17 & 14 & 40 \\
\hline S4 & 0.93 & 32 & 33 \\
\hline S6 & 1.10 & 19 & 24 \\
\hline S8 & 1.09 & 20 & 17 \\
\hline $\mathrm{S} 10$ & 1.15 & 15 & 9 \\
\hline
\end{tabular}

still falls short of reaching the total impulse obtained by the CONWEP calculations. The ALE simulations do not include the afterburning effect of the gaseous products released after the initial detonation process. Therefore, further mesh refinement above the levels used the $\mathrm{C} 10$ and the $\mathrm{S} 10$ simulations may not lead to a close match of the CONWEP calculations, which include the afterburning effects.

The lower mesh density ranges of $\mathrm{C} 1-\mathrm{C} 4$ and $\mathrm{S} 1-\mathrm{S} 4$ have deviations in the reflected overpressure and impulse in excess of $30 \%$. The higher mesh density ranges of C6-C10 and S6$\mathrm{S} 10$ are more suitable for blast simulations since the reflected overpressure and the reflected impulse are of main concern for the response of the target structure. The excessive mesh densities of the S8 and S10 simulations limit their practical usage for blast scenarios. The total number of elements in the $\mathrm{S} 6$ simulation is around 1.5 million in contrast to the 3.6 million and 7.0 million elements used in the S8 and S10 simulations, respectively. The C6 Cartesian simulation has deviations of 41 and $48 \%$ for the reflected overpressure and the reflected impulse, respectively. In contrast, the deviations for the S6 spherical simulation are 19 and $24 \%$ for the reflected overpressure and the reflected impulse, respectively. Therefore, it is concluded that the S6 spherical mesh model provides a reasonable solution without an excessive overhead of a large mesh size for blast simulations within the framework of the mesh topologies investigated in the flat plate parametric study. The S6 mesh topology is adopted in the next section for building the ALE mesh topology around the armored vehicle in the landmine blast simulation.

\section{Full-scale armored vehicle subjected to blast loads}

A full-scale experiment was conducted by the Otokar Otomotiv ve Savunma Sanayi A.S. Corporation in Turkey in 2010. The first author participated in the experiment as an observer. The purpose of the experiment was to assess the response of a Mine Resistant and Ambush Protected (MRAP) vehicle subjected to landmine blast. Figure 18 shows the experimental setup. The NATO AEP-55 Standard Vol-II [1] describes the test conditions in determining the protection level of logistic and light armored vehicles subject to grenade and blast mine threats defined by the NATO Standardization Agreement 4569. TNT is selected as the high explosive material. The landmine was placed in a steel pot buried in the ground beneath the front left tire as illustrated in Fig. 19. The steel pot has a square footprint of $700 \mathrm{~mm}$ by $700 \mathrm{~mm}$, and is $350 \mathrm{~mm}$ in height. The material used for the steel pot is $\mathrm{CrMo}_{4}$. The disc-shaped TNT charge was inserted into the circular hole in the center of the steel pot. The clearance between the TNT charge and the steel pot is $50 \mathrm{~mm}$ on the sides as well as the bottom. The TNT charge is placed into the hole such that it is suspended with the given clearance distances per the NATO AEP-55 Standard [1]. The TNT charge has a diameter to height ratio of 3:1. The purpose of the placement of the high explosive in the steel pot is to maximize reflections as set forth by the provisions of the NATO AEP-55 Standard [1].

\subsection{Experimental setup}

The primary interest of the experiment is to measure the displacements around the footrest plate and the sidewall plates of the vehicle. Displacements are measured using strain insensitive and crushable lead tubes attached on the metal plates 


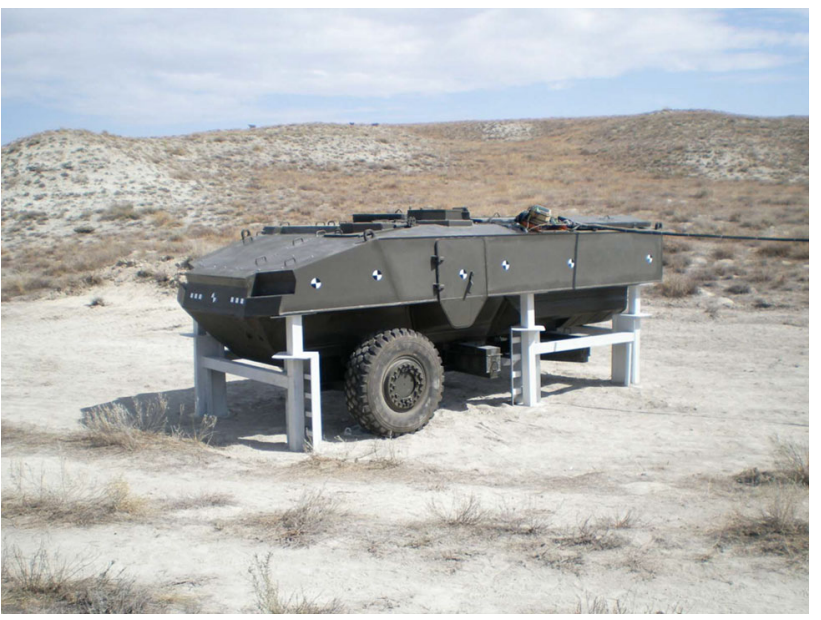

Fig. 18 Test vehicle

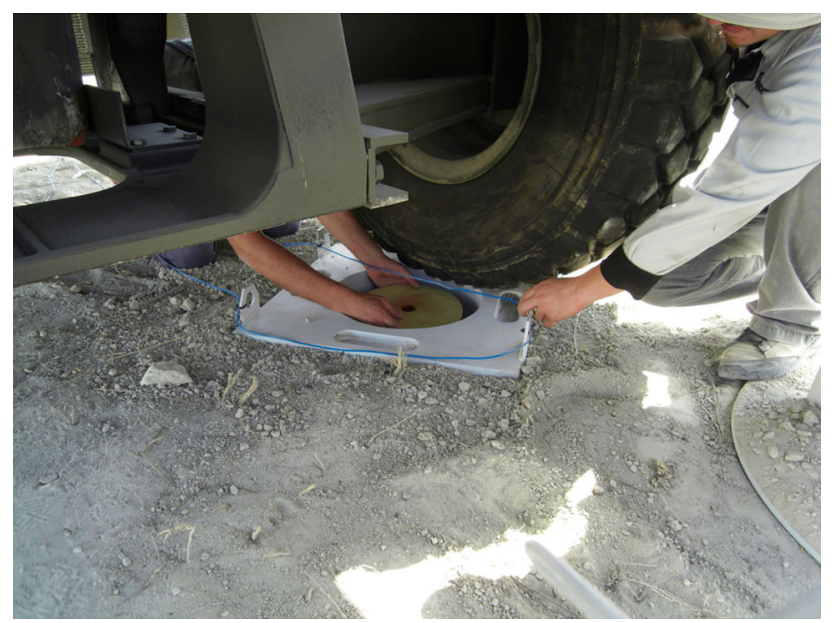

Fig. 19 Placement of the land mine in the steel pot under the front left tire

of the cabin. Figure 20 shows the status of the vehicle after explosion.

\subsection{Numerical simulation model}

The spherical mesh topology with the medium resolution of S6 is used in the simulation of the armored vehicle subjected to the landmine blast. The S6 simulation for the flat plate parametric study produced results with sufficient levels of accuracy for the arrival time of the blast wave, the blast overpressure, and the reflected impulse. The higher mesh resolutions of S8 and S10 are computationally expensive for the landmine blast simulation. Therefore, the Eulerian mesh of the S6 spherical simulation of the flat plate parametric study is used to model the air medium around the armored vehicle with 1.5 million hexahedral finite elements. The armored vehicle model consists of 316,000 shell finite elements.

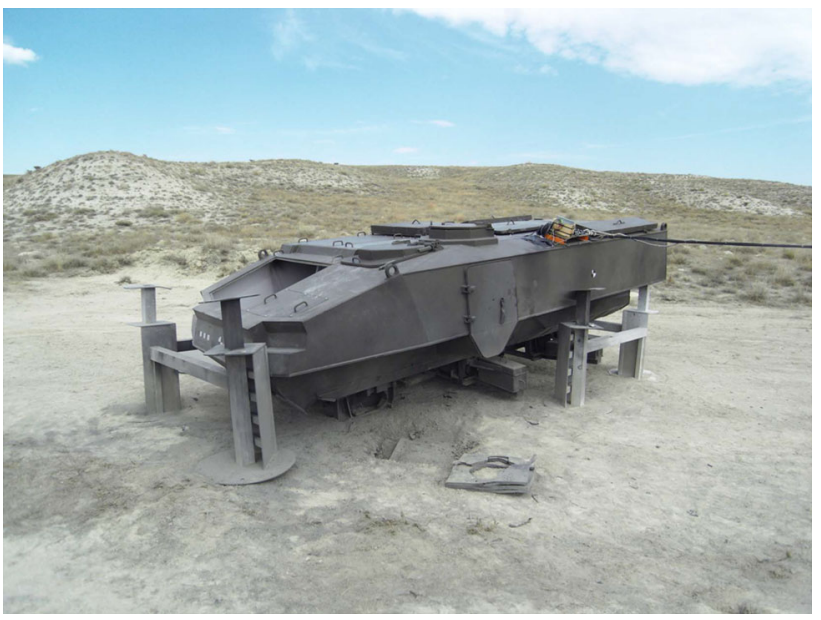

Fig. 20 Vehicle after explosion

The numerical analysis model includes the surrounding air in a hemispherical dome topology, the high explosive, and the vehicle. The landmine is placed under the front left tire. Figure 21 shows the global view of the mesh used in the simulation. The surrounding air has a diameter of $12 \mathrm{~m}$. The material model of the landmine is modeled as TNT with the JWL equation of state. The surrounding air is modeled as an ideal gas using a linear polynomial equation of state. The vehicle model contains all the crucial structural components such as the armor steel plates, the wheels, the chassis, the occupant seats, the entry doors, the door locking mechanisms, the bolts, and the welds. The entire vehicle body is modeled with Belytschko-Tsay type of shell elements [26] with five integration points. The bolts are modeled with beam elements that allow failure to occur when the load capacity is reached. A penalty-based algorithm is used to model the contact between the Lagrangian surfaces [21]. If a penetration is detected between the surfaces, equal and opposite forces proportional to the penetration depth are applied between the surfaces. The force calculation is based on the mechanical properties of the entities in contact. The finite element model in the vicinity of the front left tire is shown in Fig. 22. The TNT high explosive material and the steel pot are explicitly modeled with hexahedral elements. The non-spherical shape of the high explosive can be modeled by employing the ALE technique.

Strain rate sensitivity is taken into account in order to accurately represent the plastic behavior of steels [27]. The Johnson-Cook (J-C) [28] constitutive material model is used to model the metal components of the vehicle subjected to large strains and high strain rates. The von Mises stress of the $\mathrm{J}-\mathrm{C}$ model is given in (8) [21]:

$\sigma(\varepsilon, \dot{\varepsilon}, T)=\left[A+B \varepsilon^{n}\right]\left[1+C \ln \frac{\dot{\varepsilon}}{\dot{\varepsilon}_{0}}\right]\left[1-\left(\frac{T-T_{r}}{T_{m}-T_{r}}\right)^{m}\right]$ 


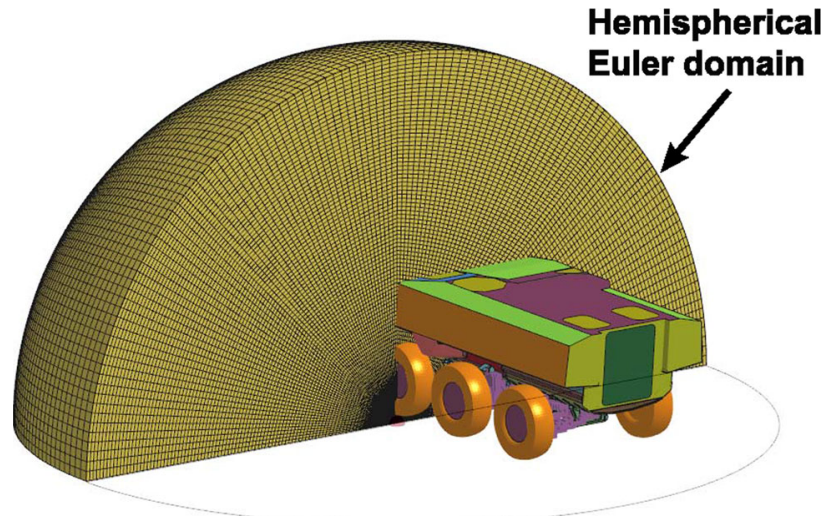

Fig. 21 Illustration of the numerical model involving the vehicle, the surrounding air, and the high explosive

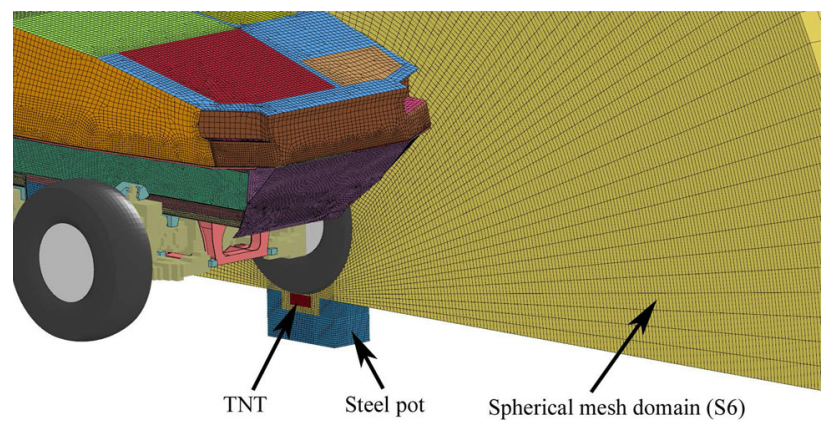

Fig. 22 Finite element mesh of the high explosive and the steel pot containment under the front left tire

where $\varepsilon$ is the equivalent strain, $\frac{\dot{\varepsilon}}{\varepsilon_{0}}$ is the dimensionless plastic strain rate, $T_{r}$ is the room temperature, and $T_{m}$ is the melting temperature. $A, B, C, n$, and $m$ are the material parameters. The vehicle body is covered with the Armox500T type of armor steel and the J-C material model parameters are obtained from the technical report FOI-R-1068-SE [29].

\subsection{Comparison between the experimental results and the simulation results}

Figure 23 demonstrates the behavior of the vehicle and the propagation of the blast waves during the 40, 200, 5500, and 7000 microseconds of the landmine blast simulation in sequence.

Figure 23a shows the detonated landmine and the resulting shock wave progressing in a hemispherical volume. Figure 23b illustrates the advance of the shock wave while it is beginning to engulf the front tire. The shock wave is redirected because of the obstruction caused by the front wheel. Figure 23c shows the flow of the blast wave between the two front tires. The coupling algorithm is able to capture the complex 3D flow phenomenon that occurs during the blast process. The blast wave progression in the Eulerian domain successfully interacts with the Lagrangian elements of the vehicle body. Figure 23d illustrates the blast waves engulfing the entire vehicle body. The regions outlined in red color illustrate the higher concentrations of the reflected overpressures.

Figure 24a provides the sensor locations inside the vehicle body. Figure $24 \mathrm{~b}$ demonstrates the comparison of the experimentally measured displacements and the results of the numerical simulation. Sensor \#8 yields the largest displacement and is reported in Fig. 24b with a normalized value of 100. Displacements at the other sensor locations are provided as a fraction of the normalized value at sensor \#8. In the post-test evaluation of the vehicle compartment, it was observed that the coupling links of the displacement sensors at locations \#1 and \#10 slipped during the landmine blast.

Figure $24 \mathrm{c}$ shows the deviation percentages of the simulation results with respect to the experimental measurements for each displacement sensor. The largest discrepancies occurred at the sensor locations \#1 and \#10. The deviation in the blast simulation is less than $25 \%$ for all other sensor locations. It was observed after the experiment that the measurement tubes for monitoring the displacements separated from the sidewall during the blast process at the sensor locations \#1 and \#10. This fact may have caused an impact of the measurement tubes with the sidewalls of the vehicle, resulting in inaccurate measurements of the displacements. The LS-Dyna simulation does not include the afterburning effects in terms of the gaseous products of the detonation reacting with the ambient oxygen in the surrounding air medium. As a consequence the release of the afterburning energy is not modeled in the TNT type of high explosive landmine simulation. However, the displacements calculated in the LS-Dyna simulation matched well with the experimental measurements. The simulation run-time on the Windows HPC Server 2008 computing cluster utilizing 36 processors is 42,240 seconds.

\section{Conclusions}

Mesh topology and resolution significantly affect the results of a blast simulation. The use of a coarse mesh resolution yields inaccurate results in the computational fluid dynamics calculations of the blast wave in the Eulerian domain. When the vehicle body is discretized using a fine mesh resolution, the shell finite elements of the Lagrangian domain govern the critical time step of the explicit time integration and significantly increase the computational expense. Therefore, a combination of fine Eulerian mesh resolution and coarse Lagrangian mesh resolution is optimal for the ALE modeling approach used in blast simulations. The main goal of the ALE model is to obtain accurate solutions for the reflected overpressure and impulse. 
Fig. 23 Illustration of the progress of the blast wave at various instances of the numerical simulation

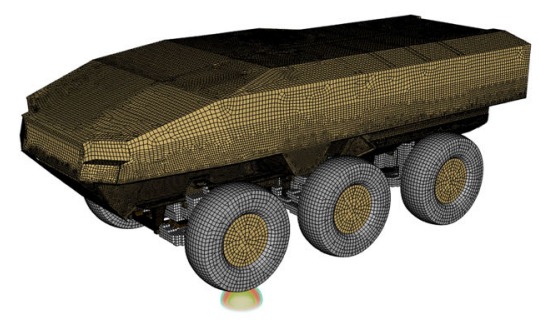

(a) $40 \mu \mathrm{s}$

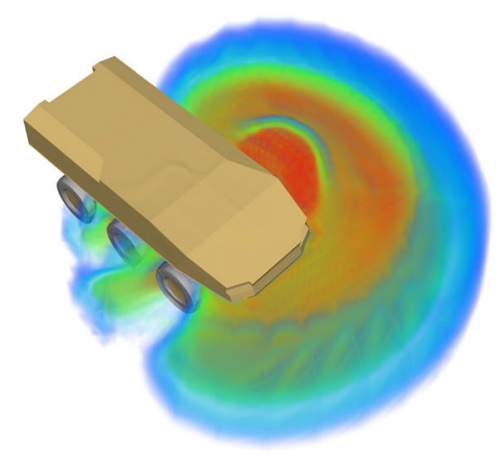

(c) $5500 \mu \mathrm{s}$

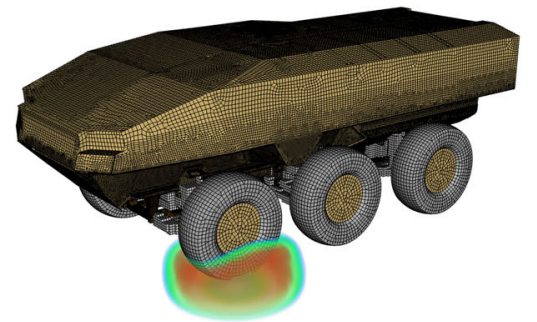

(b) $200 \mu \mathrm{s}$

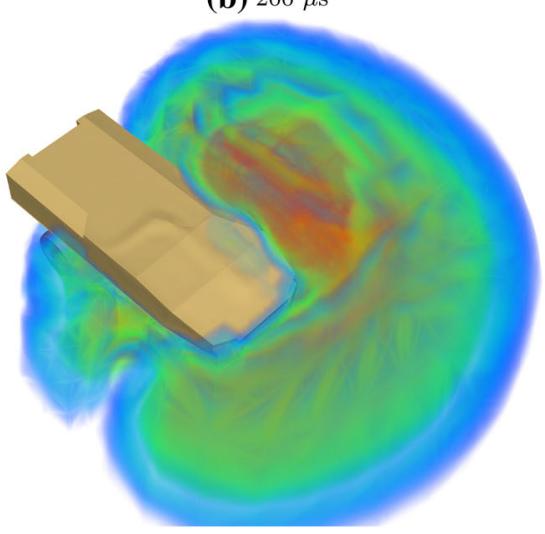

(d) $7000 \mu \mathrm{s}$

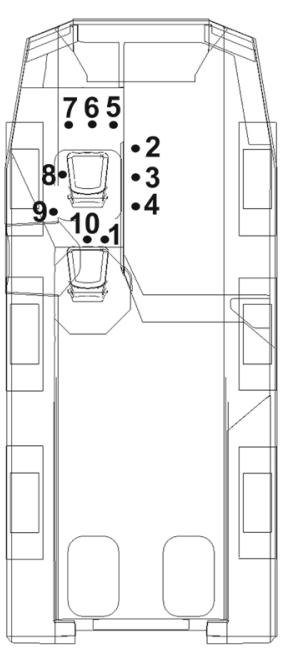

(a)

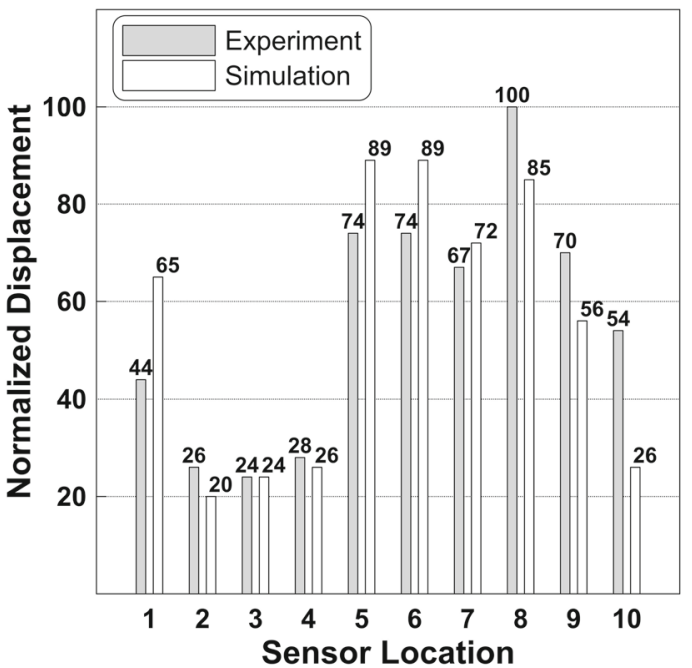

(b)

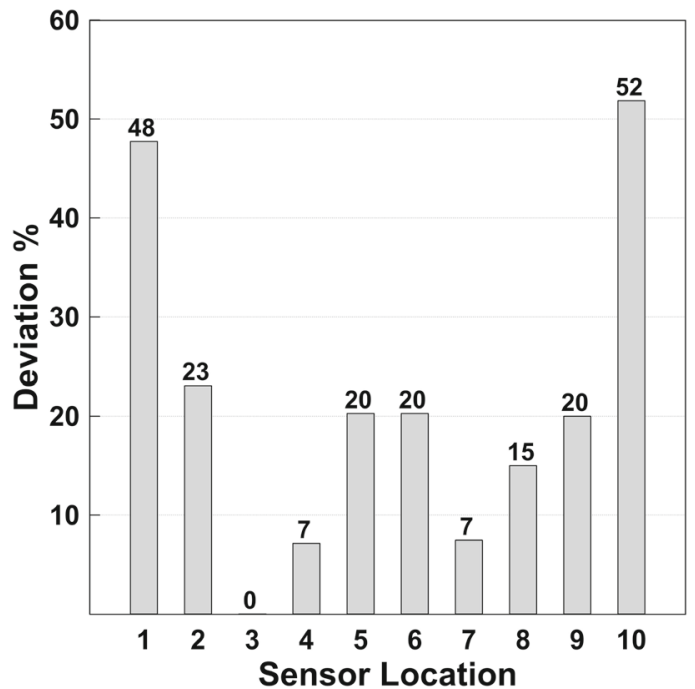

(c)

Fig. 24 a Sensor locations inside the vehicle cabin (top view), b normalized displacement values, c deviation of the simulation results with respect to the experimental measurements

The Cartesian topology is commonly used in the Eulerian domain of blast simulations. The spherical topology provides an alternative solution and may provide better results for the reflected overpressure and impulse.

A parametric study of a rigid reflecting surface subjected to a point charge is investigated in this study in order to determine the effects of mesh resolution and topology for the incident overpressure, the incident impulse, the reflected overpressure, and the reflected impulse. The results are compared with the experimentally verified CONWEP calculations. The Cartesian topology underestimates both the reflected overpressure and impulse even when higher mesh resolutions are used. However, the use of a spherical topology leads to sufficient accuracy in terms of the reflected overpressure and impulse. The shock wave arrival time is also better estimated in the simulations using the spherical topology.

An experimental study was conducted on an armored personnel carrier in 2010. A landmine was placed under the front 
left tire. The results of the experiment are compared with the blast simulation presented in this study. The rigid reflecting surface parametric study shows that accurate results can be obtained for the S6 spherical topology simulation with a level of medium-to-higher mesh resolution. Therefore, the Lagrangian and Eulerian domains of the armored personnel carrier are meshed with a spherical mesh topology similar to the S6 case of the rigid reflecting surface parametric study. The comparison of the experimentally measured displacements at various locations around the region of interest shows good agreement with the ALE blast simulation results.

Acknowledgments The authors would like to thank the Technology and Innovation Funding Programs Directorate (TEYDEB) of TUBITAK (Turkish Scientific and Technological Research Council) for the major financial support of the research project provided through grant no. 3060493, and the OTOKAR Otomotiv ve Savunma Sanayi A.S. Corporation. The second author gratefully acknowledges the funding support provided by TUBITAK through Grant No. 107M002.

Open Access This article is distributed under the terms of the Creative Commons Attribution 4.0 International License (http://creativecomm ons.org/licenses/by/4.0/), which permits unrestricted use, distribution, and reproduction in any medium, provided you give appropriate credit to the original author(s) and the source, provide a link to the Creative Commons license, and indicate if changes were made.

\section{References}

1. N.A.T.O.: Procedures for evaluating the protection level of logistic and light armoured vehicles-Mine Threat, $1^{\text {st }}$ edn. Report AEP-55, vol. 2. Allied Engineering Publication (2006)

2. Chafi, M.S., Karami, G., Ziejewski, M.: Numerical analysis of blast-induced wave propagation using FSI and ALE multi-material formulations. Int. J. Impact Eng. 36(10-11), 1269-1275 (2009)

3. Slavik, T.P.: A coupling of empirical explosive blast loads to ALE air domains in LS-DYNA. In: 7th European LS-DYNA Conference, Salzburg, pp. 1-10 (2009)

4. Kwasniewski, L., Balcerzak, M., Wojciechowski, J.: A feasibility study on modeling blast loading using ALE formulation. In: Cost Action C26: Urban habitat constructions under catastrophic events, Naples, pp. 127-132 (2010)

5. Kilic, S.A.: ALE mesh sensitivity simulations for blast perimeter walls in protective structural engineering. In: CMM-2011: 19th International Conference on Computer Methods in Mechanics, Warsaw University of Technology, Poland, pp. 243-244 (2011)

6. Kilic, S.A., Smith, P.D.: Behaviour of deformable blast walls for protective structural design. In: COST Action C-26: Urban habitat constructions under catastrophic events, Naples, pp. 477-480 (2010)

7. Kilic, S.A., Smith, P.D.: Simulation of pressures behind rigid blast walls. In: COST Action C-26: Urban habitat constructions under catastrophic events, Naples, pp. 481-486 (2010)

8. Erdik, A., Kilic, N., Guden, M., Tasdemirci, A.: Numerical approach to design process of armored vehicles. In: Proceedings of the ASME: 10th Biennial Conference on Engineering Systems Design and Analysis, Istanbul, vol. 4, pp. 231-237, (2010)

9. Yin, X., Gu, X., Lin, F., Kuang, X.: Numerical analysis of blast loads inside buildings. In: Proceedings of the International Symposium on Computational Structural Engineering, Shanghai, pp. 681-690 (2009)
10. Zakrisson, B., Wikman, B., Haggblad, H.A.: Numerical simulations of blast loads and structural deformation from near-field explosions in air. Int. J. Impact Eng. 38(7), 597-612 (2011)

11. Departments of the Army, the Navy, and the Air Force, Structures to resist the effects of accidental explosions. Report TM 5-1300/NAVFAC P-397/AFR 88-22, Washington, DC (1990)

12. Hyde, D.W.: User's guide for microcomputer programs CONWEP and FUNPRO, applications of TM 5-855-1, 'Fundamentals of protective design for conventional weapons'. Report SL-88-1, U.S. Army Corps of Engineers Waterways Experiment Station, Vicksburg, MS (1988)

13. Kingery, C., Bulmash, G.: Air-blast parameters from TNT spherical air burst and hemispherical surface burst. Report ARBRL-TR02555, Ballistic Research Laboratory, Aberdeen Proving Ground, Aberdeen, MD (1984)

14. Randers-Pehrson, G., Bannister, K.A.: Airblast loading model for DYNA2D and DYNA3D. Report ARL-TR-1310, U.S. Army Research Laboratory, Aberdeen Proving Ground, MD (1997)

15. Hallquist, J.: LS-DYNA keyword user's manual, vol. 1. Livermore Software Technology Corporation, Livermore (2012)

16. Formby, S.A., Wharton, R.K.: Blast characteristics and TNT equivalence values for some commercial explosives detonated at ground level. J. Hazard. Mater. 50, 183-198 (1996)

17. Wharton, R.K., Formby, S.A., Merrifield, R.: Airblast TNT equivalence for a range of commercial blasting explosives. J. Hazard. Mater. A79, 31-39 (2000)

18. Alonso, F.D., Ferradas, E.G., Perez, J.F.S., Aznar, A.M., Gimeno, J.R., Alonso, J.M.: Characteristic overpressure-impulse-distance curves for the detonation of explosives, pyrotechnics or unstable substances. J. Loss Prevent. Proc. 19, 724-728 (2006)

19. Dobratz, B.M., Crawford, P.C.: LLNL explosives handbook: Properties of chemical explosives and explosive simulants. Report UCRL-52997, Lawrence Livermore National Laboratory, California (1981)

20. Kinney, G., Graham, K.: Explosive shocks in air, vol. 1. Springer, New York (1985)

21. Hallquist, J.: LS-DYNA Theory manual. Livermore Software Technology Corporation, Livermore (2006)

22. Neuscamman, S., Pezzola, G., Alves, S., Glenn, L., Glascoe, L.: Incorporating afterburn effects into a fast-running tool for modeling explosives in tunnels. Report UCRL-CONF-599056, Lawrence Livermore National Laboratory, California (2012)

23. Balakrishnan, K., Genin, F., Nance, D.V., Menon, S.: Numerical study of blast characteristics from detonation of homogeneous explosives. Shock Waves 20, 147-162 (2010)

24. Togashi, F., Baum, J.D., Mestreau, E., Löhner, R., Sunshine, D.: Numerical simulation of long-duration blast wave evolution in confined facilities. Shock Waves 20, 409-424 (2010)

25. Zukas, J.A., Walters, W.P.: Explosive effects and applications. Springer, New York (2003)

26. Belytschko, T.B., Lin, J.I., Tsay, C.S.: Explicit algorithms for the nonlinear dynamics of shells. Comput. Methods Appl. Mech. Eng. 42(2), 225-251 (1984)

27. Jones, N.: Structural Impact. Cambridge University Press, Great Britain (1989)

28. Johnson, G.R., Cook, W.H.: A constitutive model and data for metals subjected to large strains, high strain rates and high temperatures. In: Seventh International Symposium on Ballistics, The Hague, The Netherlands, pp. 541-547 (1983)

29. Nilsson, M.: Constitutive model for Armox 500T and Armox 600T at low and medium strain rates. Report FOI-R-1068-SE, Swedish Defence Research Agency (2003) 